\title{
EL CONFLICTO EN LA APLICACIÓN DE LA NORMA TRIBUTARIA EN LAS OPERACIONES DE REESTRUCTURACIÓN EMPRESARIAL
}

\author{
Miguel GutiÉRREZ BengoeCHEA \\ Profesor Titular de Derecho Financiero y Tributario \\ mgutierrez@uma.es \\ Universidad de Málaga
}

\begin{abstract}
Resumen
El proceso de globalización económica en el que están inmersas todas las economías del mundo conlleva que las grandes empresas aprovechen los resquicios legales para desarrollar estrategias fiscales agresivas colocando el capital en territorios calificados como paraísos fiscales con el único fin de conseguir un ahorro fiscal en sus obligaciones tributarias. En estos casos, se utiliza directamente la evasión fiscal pero, en otros casos, se utilizan otros expedientes jurídicos como la elusión fiscal que en el Derecho tributario español toma cuerpo jurídico a través del conflicto en la aplicación de la norma tributaria.

A nivel internacional, la normativa tributaria española inspirada en Directivas europeas regula el concepto de motivo económico válido para determinar en qué situaciones, las operaciones de reestructuración empresarial, crean un conflicto en la aplicación de la norma $\mathrm{y}$, por tanto, se presumen que están realizadas con el único objetivo de obtener un ahorro fiscal.
\end{abstract}

Palabras claves: Conflicto; Fraude; Globalización; Válido; Impuesto

\begin{abstract}
:
The process of economic globalization which involves all world economies enables big compañies to take advantage of legal gaps to develop aggressive tax strategies placing capital in tax heavens (Paradises) with the only purpose to reduce their tax liabilities. In such cases tax evasion is used directly, but, on the other hand, legal instruments such as tax avoidance are also used, which, according to Spanish tax law, take the legal form due to the conflict in the application of tax rule.

At the international level, Spanish tax regulation based on EU Directives, regulate what forms of business restructuring operations are valid, and which create conflict with the application of the rule and are therefore assumed to be performed with the sole objective to minimize taxes.
\end{abstract}

Key words: Conflict, Fraud, Globalization, Valid, Tax

\section{Sumario:}

1. Introducción. 2. Cláusula general versus cláusula específica en la prevención del conflicto en la aplicación de la norma tributaria. 3. El conflicto en la aplicación de la norma tributaria versus simulación y economía de opción. 4. El conflicto en la aplicación de la norma tributaria y su calificación tributaria. 4.1. Obtención de una ventaja económica. 4.2. Infracción tributaria en el conflicto en la aplicación de la norma tributaria. 5. El motivo económicamente válido en las operaciones de reestructuración empresarial. 6. Conclusiones

REJIE Nueva época: Revista Jurídica de Investigación e Innovación Educativa

Núm.17, Enero 2018, pp. 127-139

[En línea] http://www.revistas.uma.es/index.php/rejie 


\section{Introducción}

El fraude de Ley en el Derecho tributario es un instituto jurídico que implica un abuso de las formas jurídicas por parte de los contribuyentes con el único fin de obtener un ahorro fiscal.

Para la consecución de este objetivo fiscal los obligados tributarios utilizan unas maniobras jurídicas que terminan en un abuso de las formas jurídicas.

Desde un punto de vista económico el sujeto realiza una declaración tributaria completa a la Administración tributaria pero eludiendo la norma tributaria que debiera aplicarse y utilizando una norma de cobertura con el único propósito de obtener una ventaja fiscal.

Hasta la reforma de la Ley General Tributaria (en adelante LGT) el fraude de Ley tributario coincidía con el conflicto en la aplicación de la norma tributaria, sin embargo, con la modificación de la LGT no hay una coincidencia entre ambos institutos jurídicos.

En colación a lo anterior, la reforma de la LGT apunta al especial ánimus del obligado tributario a cometer un conflicto en la aplicación de la norma tributaria ya que el hecho de realizar un negocio jurídico de forma artificiosa excluye la aplicación razonable de la norma tributaria. Este hecho se traduce en que es posible que en el conflicto en la aplicación de la norma tributaria la conducta del obligado tributario sea sancionada.

El artículo 15 de la modificación de la LGT mantiene en sus dos primeros apartados la misma redacción que la Ley 53/2003, por lo que persiste la cláusula general anti-abuso al derecho tributario. Este precepto es similar al fraude de ley que regulaba el artículo 24 de la LGT de 1963; sin embargo, actualmente goza de mayor flexibilización y es de más fácil aplicación por varios motivos. El primero de ellos surge porque de la redacción del artículo 24 de la LGT de 1963, se dejaba abierta la posibilidad a una aplicación analógica de la norma reguladora del hecho imponible, por otro lado, había que tener en cuenta el aspecto subjetivo del obligado tributario en la comisión del fraude tributario, es decir, la Administración tenía que demostrar el propósito de eludir del obligado tributario, por lo que en muchas ocasiones, se convertía en una probatio diabólica para el órgano tributario ${ }^{1}$.

Ambos institutos pretenden combatir la elusión fiscal atendiendo a la causa que rige en los negocios jurídicos y aplicando posteriormente el principio de calificación jurídica regulado en el artículo 13 de la $\mathrm{LGT}^{2}$. Con esta combinación de preceptos tributarios es posible detectar la verdadera causa que rige en los contratos típicos o atípicos establecidos por las personas físicas o jurídicas con independencia de la forma que subjetivamente hayan adoptados las partes.

\footnotetext{
${ }^{1}$ Por esta razón apenas se iniciaban expedientes de fraude de ley en el ámbito tributario, sobre todo, porque obliga a los órganos de inspección a aplicar normas de carácter procesal, es decir, presunciones basadas muchas veces en simples indicios con los problemas que podrían suscitarse en relación al principio de seguridad jurídica y proporcionalidad.

${ }^{2}$ El artículo 13 LGT dispone que: "Las obligaciones tributarias se exigirán con arreglo a la naturaleza jurídica del hecho, acto o negocio realizado, cualquiera que sea la forma o denominación que los interesados le hubieran dado y prescindiendo de los defectos que pudieran afectar a su validez".
} 
Esta última consideración nos lleva a reiterar la mayor flexibilidad con la que es posible aplicar la cláusula general regulada en el artículo 15 de la LGT ya que, a diferencia de la anterior regulación, no se tiene que demostrar el aspecto subjetivo, en la elusión total o parcialmente del hecho imponible con el único objetivo de conseguir un ahorro fiscal.

\section{Clausula general versus clausula especifica en la prevención del conflicto en la aplicación de la norma tributaria}

Las clausulas generales anti-abuso tienen como fin principal disuadir determinados comportamientos tendentes al fraude de ley. En nuestro ordenamiento tributario es el artículo 15 de la LGT el que regula la cláusula general anti-abuso del derecho, caracterizándose por su gran generalidad dado que sus efectos se extienden a todo el sistema tributario, pudiendo ser aplicado frente a cualquier tributo y ante cualquier acto o negocio realizado por un obligado tributario ${ }^{3}$.

Las clausulas generales suelen ser abstractas y generales lo que en principio tiene efectos positivos y negativos en las partes de la relación jurídica tributaria. Por un lado, la Administración siempre podrá aplicar la cláusula general de prevención del fraude de ley a situaciones en que la Administración tributaria considere que se ha producido un conflicto en la aplicación de la norma tributaria ${ }^{4}$. Respectos a sus posibles efectos negativos, con este tipo de cláusula que el legislador introduce en las normas tributarias se pierde seguridad jurídica porque los contribuyentes desconocen a qué supuestos en concreto se aplicaría la norma anti-fraude.

Para facilitar la aplicación del artículo 15 de la LGT hay que basarse en criterios objetivos y gozar de la suficiente flexibilidad para contrarrestar, en su caso, conceptos jurídicos indeterminados que a veces la integran. Sobre todo, porque en la fase probatoria corresponde a la Administración determinar que se dan los presupuestos aplicativos de la cláusula general anti-fraude.

No obstante, el concepto de conflicto en la aplicación de la norma es más complejo y para prevenirlo el legislador tributario regula cláusulas antifraude específicas que desalientan posibles actuaciones que probablemente desembocarían en situaciones de conflicto en la aplicación de la norma tributaria. Este es el caso del régimen especial de diferimiento de las plusvalías en el Impuesto sobre Sociedades en el que el legislador advierte que debe haber un motivo económico válido que justifique la aplicación de dicha forma de tributar. Esta cláusula específica no es trasladable a otros actos de tipo empresarial regulados en el Impuesto sobre Sociedades- salvo regulación expresa-, aunque exista una identidad sustancial con otros negocios jurídicos cuyo hecho imponible pertenezcan a otra figura tributaria, porque de ser así, se podría incurrir en la analogía.

\footnotetext{
${ }^{3}$ Dado el amplio campo de aplicación que tiene la cláusula general anti-fraude, ésta tiene que estar sometida al principio de legalidad.

${ }^{4}$ En este sentido, con la cláusula general de prevención del fraude de ley se garantiza el efecto disuasorio al venir fijado por una norma de rango legal y garantizar la eficacia de la norma a situaciones ex-ante así como ex -post en ejecución de la misma.
} 
Las cláusulas específicas toman normalmente cuerpo jurídico en normas sustantivas como las ficciones en las que es frecuente que lleven interrelacionadas alguna norma procesal como son las presunciones tributarias. Esta consideración hace que estas normas faciliten la prueba a la Administración a la vez que son inatacables procesalmente.

Es muy común que las clausulas específicas de prevención del fraude de ley no realicen ninguna actuación específica para combatir la elusión fiscal, sino simplemente se aplica la norma reguladora del hecho imponible del tributo ${ }^{5}$. Por tanto, la existencia de una cláusula específica en la norma tributaria es ejecutable directamente siempre que se den los presupuestos contenidos en el precepto que la regula. Todo lo anterior, nos lleva a dos consideraciones. En primer lugar, existe un criterio de preferencia a aplicar la norma tributaria ad hoc sobre la de carácter general. En segundo lugar, cuando las normas específicas anti abuso son de naturaleza sustantivas y, por lo tanto, constituyen "per se" una verdad normativa excluyen el planteamiento de un conflicto en la aplicación de la norma ${ }^{6}$. Esta situación difiere sustancialmente cuando la norma específica para evitar la elusión fiscal se articula a través de normas procesales como son las presunciones iuris tantum.

En estos supuestos la norma no contiene una verdad sustantiva o material, lo que da oportunidad al obligado tributario a que accione frente a la norma procesal para satisfacer sus pretensiones ${ }^{7}$.

\section{El conflicto en la aplicación de la norma tributaria versus simulación y economía de opción}

Reiterando en parte, lo expuesto anteriormente, el conflicto en la aplicación de la norma tributaria está regulado en el artículo 15 de la LGT que en su literalidad dispone que "Se entenderá que existe conflicto en la aplicación de la norma tributaria cuando se evite total o parcialmente la realización del hecho imponible o se minore la base o la deuda tributaria mediante actos o negocios en los que concurran las siguientes circunstancias:

a) Que, individualmente considerados o en su conjunto sean notoriamente artificiosos o impropios para la consecución del resultado obtenido.

b) Que de su utilización no resulten efectos jurídicos o económicos relevantes, distintos del ahorro fiscal y de los efectos que se hubieran obtenido con los actos o negocios usuales o propios".

Desde el derecho privado este precepto de prevención general del fraude de ley se aplica cuando la autonomía de la voluntad que tienen las personas para organizar y gestionar su patrimonio, rebasan los cauces formales establecidos en el Derecho

\footnotetext{
${ }^{5}$ Así, por ejemplo, en el Impuesto sobre Transmisiones Patrimoniales se establece una norma anti-abuso en la problemática del pago de pensiones privadas a personas físicas a cambio de la entrega de la vivienda a otra persona física para evitar enriquecimientos injustos en el pagador de la pensión.

${ }^{6}$ Cfr HERRERA MOLINA, PM. (1999) "Nuevas perspectivas del fraude de ley en materia tributaria" Quincena Fiscal, pág 14.

${ }^{7}$ En el IRPF se establece la presunción iuris tantum sobre la residencia en territorio español de la persona física que resida en el extranjero cuando su cónyuge e hijos menores residan en España.
} 
privado $^{8}$. En este sentido y a tenor del artículo 1255 del Código civil en el que dispone que "los contratantes pueden establecer pactos, cláusulas y condiciones que tengan por convenientes siempre que no sean contrarias a las leyes, a la moral ni al orden público", los obligados tributarios pueden planificar fiscalmente sus patrimonios para disminuir su factura fiscal dentro de las posibilidades que ofrecen las normas dispositivas del derecho tributario.

En estos casos, en el que el obligado tributario aplica la norma más favorable fiscalmente está realizando lo que en el Derecho tributario se denomina economía de opción.

En realidad cuando el sujeto pasivo opta por aplicar normas privadas para conseguir principalmente una ventaja fiscal está desarrollando una economía de opción, sólo que por separarse de los cauces formales y de las instituciones jurídicas preestablecidas en el derecho privado para conseguir tales fines, entonces se considera como una economía de opción no permitida en el Derecho tributario. En este supuesto es cuando tiene aplicación el conflicto en la aplicación de la norma tributaria.

Llama la atención en la definición de conflicto en la aplicación de la norma tributaria cuando especifica que se entiende originado el conflicto en la aplicación de la norma cuando se evite total o parcialmente el hecho imponible. No tiene mucho sentido, el no realizar parcialmente el hecho imponible, pues el hecho imponible es único en función de unos presupuestos de naturaleza jurídica que establece la norma tributaria ${ }^{9}$.

No obstante, hay similitudes en ambos conceptos que a veces es complicado discernir cuando acaba la economía de opción y cuando empieza el conflicto en la aplicación de la norma tributaria. Tanto en el conflicto en la aplicación de la norma tributaria como en la economía de opción existe una concordancia entre lo formalizado y la realidad. Sin embargo, la economía de opción no atenta a lo querido en el espíritu de la ley, mientras que en conflicto en la aplicación de la norma tributaria si se produce ${ }^{10}$.

La simulación es regulada en el artículo 16.1 LGT el cual dispone que "En los actos o negocios en los que exista simulación, el hecho imponible gravado será el efectivamente realizado por las partes".

Como la norma básica en la ordenación de los tributos sigue sin definir la simulación a efectos tributarios se entiende que hay que identificarla con la simulación regulada en el Derecho civil, según el cual, se crea una apariencia con un fin determinado, aunque precisamente ello suponga que sea declarada falsa la causa en el negocio simulado. Según la doctrina en la simulación deben concurrir dos elementos: en primer lugar debe haber un acuerdo entre quien emite la declaración y quien la recibe de que la misma no

\footnotetext{
${ }^{8}$ Cfr PÉREZ ROLLO, F y AGUALlO AVILÉS, A (1996) . Comentarios a la reforma de la Ley General Tributaria; Aranzadi; pág 47. Estos autores nos recuerdan que en el fraude de Ley no hay una violación directa del ordenamiento; el objetivo de conseguir un ahorro fiscal se alcanza precisamente aprovechando las vías ofrecidas por las normas jurídicas aunque utilizadas de manera distintas a su espíritu.

${ }^{9}$ Cfr CALVO VERGEZ ,J.(2007) "Cláusula antiabuso versus fraude de ley: ¿dos conceptos semejantes en la Ley General Tributaria?" Crónica Tributaria; $n^{\circ} 123$; pág 25

${ }^{10}$ Cfr PALAO TABOADA; C (1998). "Existe fraude a la ley tributaria" Revista de Tributación y Contabilidad, $\mathrm{n}^{\circ}$ 182; pág 13. Para que haya una verdadera economía de opción el negocio realizado tiene que reunir no sólo los requisitos formales sino también los sustanciales del correspondiente tipo negocial.
} 
será eficaz para producir efectos vinculantes. En segundo lugar, es esencial la finalidad de engaño, haciendo creer a los terceros en la realidad de un negocio que no existe.

Hay que destacar que la redacción del artículo 16 LGT se refiere literalmente al hecho imponible y la liquidación ${ }^{11}$. Sin embargo, cabe entender que la simulación puede alcanzar a cualquier obligación tributaria y dentro de la obligación tributaria principal, a cualquier elemento de la misma. Más problemático resulta que la regulación del artículo 16 LGT abarque actuaciones que no terminan en liquidación como por ejemplo, las actuaciones recaudatorias, ya que tradicionalmente se ha entendido que en el procedimiento de apremio no se puede apreciar la existencia de simulación por la Administración y es necesario acudir previamente a los Tribunales de Justicia ${ }^{12}$.

Aunque no hay una clasificación expresa de las clases de simulación tributaria podemos identificarla en dos tipos en relación a la intensidad o gravedad del negocio simulado.

En primer lugar hay que destacar la simulación absoluta que se produce cuando no existe el negocio simulado, pero tampoco existe otro negocio real que apoye al simulado.

La finalidad normal de este tipo de simulación es defraudar a los acreedores, bien fingiendo una disminución del activo o bien aparentando un aumento del pasivo.

Existe simulación relativa, cuando las personas declaran un negocio jurídico que no es el que realmente no desean perfeccionar. El que las partes declaran a efectos tributarios se denomina negocio simulado y el realmente querido es el disimulado. A efectos ilustrativos, un caso simple de simulación relativa sucede cuando dos personas físicas realizan un contrato de compraventa de una vivienda a un precio irrisorio y entre ellas existe un lazo de parentesco. En este supuesto, ambas partes esconden el verdadero negocio jurídico, en este caso, una donación y muestran a la Administración tributaria una compraventa. La diferencia de tributación entre ambos impuestos es bastante significativa.

Normalmente, este tipo de instituto jurídico trasladado al Derecho fiscal puede recaer en determinados elementos de la estructura del tributo como puede ser el consentimiento, objeto e incluso puede también afectar a elementos subjetivos como puede ser la interposición ficticia de una persona.

En mi opinión, el artículo 16 LGT es un supuesto específico de calificación cuando se produce la simulación. En este sentido, cuando las partes que intervienen en el negocio jurídico utilizan la simulación la Administración puede recalificarlo y adecuarlo a la realidad pretendida $\mathrm{y}$, en su caso, a la legalidad, en los supuestos de simulación absoluta.

\footnotetext{
${ }^{11}$ En este sentido el artículo 16.2 LGT dispone que: "La existencia de simulación será declarada por la Administración tributaria en el correspondiente acto de liquidación, sin que dicha calificación produzca otros efectos que los exclusivamente tributarios".

${ }^{12}$ Vid DRRN 27/9/2003.
} 
Sin embargo, hay que diferenciar la calificación jurídica que se produce en todo caso, cuando existe simulación tributaria de aquellos casos, en que existe una calificación defectuosa por parte de los contribuyentes ${ }^{13}$.

A tenor del artículo 16.2 LGT la simulación será declarada por la Administración tributaria en el correspondiente acto de liquidación. Aunque lo habitual es que se declare en el procedimiento de inspección tributaria no hay preceptos que impidan que se declare la simulación en un procedimiento de comprobación ${ }^{14}$.

También podríamos analizar la diferenciación entre ambos conceptos jurídicos estudiando la causa de los contratos ya que ésta opera como un medio de control de la legalidad de los negocios jurídicos. En este sentido, la verdadera naturaleza del negocio jurídico no tendrá por qué coincidir con los aspectos subjetivos de las partes que determinan la forma jurídica de los negocios sino con la causa perseguida por las partes intervinientes en el negocio jurídico. En los supuestos en los que no exista una concordancia entre el tipo de negocio elegido por las partes con la verdadera naturaleza de dicho negocio habría que aplicar el principio de calificación para determinar la verdadera naturaleza jurídica del negocio realizado ${ }^{15}$.

\section{EI conflicto en la aplicación de la norma tributaria y su calificación jurídica}

Llegado a este punto se suscitan algunas consideraciones como si es posible aplicar por los órganos de inspección el principio de calificación de forma autónoma sin acudir al artículo 15 de la LGT cuando pretenda corregir la elusión fiscal o simplemente dentro del procedimiento que conlleva el conflicto en la aplicación de la norma tributaria, los órganos de inspección harían uso del principio de calificación tributaria. La cuestión planteada se resuelve a favor de la última consideración, es decir, una vez que se inicie el procedimiento tributario para detectar la norma eludida el órgano inspector podrá aplicar el principio de calificación tributaria junto a los demás elementos probatorios que en la fase de indagación e investigación obtenga la Administración tributaria. La tesis de aplicar directamente el principio de calificación jurídica además de dejar sin aplicación jurídica al artículo 15 de la LGT nos encontraríamos ante un supuesto de nulidad de pleno derecho del artículo 217.1.e) LGT pues el acto habría sido dictado prescindiendo total y absolutamente del procedimiento legalmente establecido.

\subsection{Obtención de una ventaja económica}

\footnotetext{
${ }^{13}$ Habría una defectuosa calificación tributaria si un contribuyente ha considerado como gasto deducible en el rendimiento del capital inmobiliario los gastos de mejora, confundiéndose con los gastos de reparación y conservación. Sin embargo, si el contribuyente ha firmado un contrato con una empresa inexistente especializada en reparaciones con el sólo propósito de justificar y deducir los gastos de reparación y conservación, habría que acudir al expediente del artículo 16 LGT.

${ }_{14}^{14}$ Este aspecto diferencia la simulación en el derecho del conflicto en la aplicación de la norma tributaria.

${ }^{15}$ Cfr PALAO TABOADA, C" Existe el fraude a la ley tributaria” op cit, pgs 7-8, en el citado trabajo se expresa que los negocios jurídicos habrán de calificarse a la luz de las normas sustantivas aplicables, generalmente civiles o mercantiles y si la ley fiscal utiliza para definir el hecho imponible de un tributo el nombre de un determinado tipo de negocio o contrato, hay que entender, en principio, que lo utiliza en el sentido que tiene en la rama del Derecho correspondiente.
} 
El artículo 15 de la LGT en su segundo apartado establece el segundo requisito que debe cumplirse para plantear el conflicto en la aplicación de la norma tributaria. En este sentido, el citado apartado dispone que "no resulten efectos jurídicos o económicos relevantes distintos del ahorro fiscal y de los efectos que se hubieran obtenido con los actos o negocios usuales o propios". Esta segunda parte del precepto marca el aspecto finalista del conflicto en la aplicación de la norma tributaria que se reduce fundamentalmente al ahorro fiscal.

El concepto de ahorro de impuestos deviene por una minoración de la cuota tributaria, fruto de la aplicación de una norma tributaria más favorable que, bien porque contiene una serie de beneficios fiscales o porque en sí misma, actúa sobre la base imponible o sobre el tipo de gravamen originando como efecto final, la reducción del coste fiscal. No obstante, el precepto en su redacción da a entender que el ahorro fiscal obtenido tiene que ser significativo en relación con los efectos jurídicos o económicos obtenidos con el negocio jurídico celebrado. En este sentido, si los efectos jurídicos obtenidos son importantes, no se aplicaría el conflicto en la aplicación de la norma, aun cuando del cumplimiento del negocio jurídico se derivase un ahorro fiscal. En cualquier caso y desde un punto de vista cuantitativo habría que ponderar el posible ahorro fiscal obtenido por las partes intervinientes en el contrato con la capacidad económica objeto de gravamen.

Por último, habría que matizar, que para que sea factible aplicar el procedimiento del conflicto en la aplicación de la norma tributaria deben concurrir de forma obligatoria los dos requisitos establecidos por el artículo 15 LGT. Esta consideración se traduce en que puede darse el caso, de que exista un vicio en la causa del contrato por abuso de las formas jurídicas y que sin embargo, no reporte una ventaja económica, en cuyo caso, no sería factible la aplicación del conflicto en la norma tributaria.

A veces; sin embargo, el concepto de conflicto en la aplicación de la norma es más complejo y para prevenirlo el legislador tributario regula cláusulas antifraude específicas, (comentadas anteriormente) que desalientan posibles actuaciones que probablemente desembocarían en situaciones de conflicto en la aplicación de la norma tributaria. Este es el caso del régimen especial de diferimiento de las plusvalías en el que el legislador advierte que debe haber un motivo económico válido que justifique la aplicación de este régimen fiscal.

\subsection{Infracción tributaria en el conflicto en la aplicación de la norma tributaria}

Hasta la última reforma de la Ley General Tributaria el conflicto en la aplicación de la norma no ha estado legalmente sancionado. Esta situación legal se mantenía por el simple hecho de que en el conflicto en la aplicación de la norma el obligado tributario realiza una declaración completa del hecho o negocio jurídico que va a realizar, por lo que no existe ocultación de elementos de patrimonio que podrían generar rentas sometidas a tributación.

No obstante, a tenor de la redacción del artículo 15.3 de la LGT el conflicto en la aplicación de la norma podría estar sancionado ya que desaparece de la redacción del precepto la negación a que sea sancionado este mecanismo de elusión de la norma tributaria.

Lo que suscita la anterior idea es en primer lugar la diferenciación del conflicto en la aplicación de la norma tributaria respecto del supuesto de fraude de Ley regulado en el 
derecho común y en segundo lugar, que el legislador se ampara en que, en el conflicto en la aplicación de la norma, no cabe el principio de interpretación razonable de la norma debido precisamente a que se realizan actos notoriamente artificiosos $\mathrm{o}$ impropios.

En este sentido el artículo 206 bis dispone que: "Constituye infracción tributaria el incumplimiento de las obligaciones tributarias mediante la realización de actos o negocios cuya regularización se hubiese efectuado mediante la aplicación del artículo 15 LGT de esta Ley y en la que hubiese resultado acreditada cualquiera de las siguientes situaciones:

a) La falta de ingreso dentro del plazo establecido en la normativa de cada tributo de la totalidad o parte de la deuda tributaria.

b) La obtención indebida de una devolución derivada de la normativa de cada tributo

c) La solicitud indebida de una devolución, beneficio o incentivo fiscal

d) La determinación o acreditación improcedente de partidas positivas o negativas o créditos tributarios a compensar o deducir en la base o en la cuota de declaraciones futuras propias o de terceros.

El incumplimiento a que se refiere el apartado anterior constituirá infracción tributaria exclusivamente cuando se acredite la existencia de igualdad sustancial entre el caso objeto de regularización y aquel o aquellos otros supuestos en que se hubiera establecido criterio administrativo y éste se hubiese publicado para general conocimiento antes del inicio del plazo para la presentación de la correspondiente declaración o autoliquidación".

Del precepto se infiere que para sancionar una conducta como infracción tributaria grave será necesario que exista un pronunciamiento administrativo y éste se haga público.

No obstante, para que exista una publicación administrativa en la que se recoja un supuesto de aplicación de la norma tributaria antes tendrá que haber un informe de la comisión consultiva tal como se regula en el artículo 15.2 de la LGT el cual dispone que:" De acuerdo con lo establecido en el artículo 15 de esta Ley, para que la inspección de los tributos pueda declarar el conflicto en la aplicación de la norma tributaria deberá emitir previamente un informe favorable de la comisión consultiva que se constituya.

La comisión consultiva está formada por 2 representantes de la DGT y por dos inspectores de hacienda.

Cuando el órgano actuante estime que puede haber un conflicto en la aplicación de la norma tributaria se le comunicará al interesado al cual se le dará unos plazos de alegaciones de 15 días.

Posteriormente el órgano de inspección actuante remitirá el expediente administrativo a la comisión consultiva.

El plazo máximo para emitir el informe es de tres meses ampliable por un mes más mediante acuerdo motivado de la comisión consultiva.

En caso de no emitirse el informe se reanudará las actuaciones inspectoras sin que desaparezca la obligación de la comisión consultiva de emitir el informe.

Este informe será vinculante para el órgano de inspección y no será susceptible de ser recurrido por el obligado tributario.

Sin embargo, las liquidaciones que procedan en base a las comprobaciones realizadas por el órgano de inspección son susceptibles de ser recurridas por los obligados tributarios". 
Del precepto transcrito se infiere que el procedimiento para la declaración del conflicto en la aplicación de la norma tributaria es laborioso y lento en su desarrollo lo que, en mi opinión, conllevará a que la Administración tributaria inicie el procedimiento cuando de los hechos comprobados se detecte un supuesto claro de conflicto en la aplicación de la norma tributaria y, sobre todo, cuando los hechos comprobados se identifiquen con otros que previamente ya han sido calificados dentro de la categoría jurídica que estamos analizando.

\section{El motivo económicamente válido en las operaciones de reestructuración empresarial}

El motivo económico válido es un concepto jurídico indeterminado de reciente incorporación al Derecho tributario español a través del derecho comunitario y que con la Directiva 90/434/CE se ha incorporado a distintas figuras impositivas como al Impuesto sobre Sociedades y al Impuesto sobre la Renta de los No Residentes ${ }^{16}$.

Este concepto aparece ligado como cláusula general de prevención del conflicto en la aplicación de la norma tributaria, sobre todo, en operaciones internacionales pero, que también, como se ha expuesto, como cláusula específica en determinadas figuras tributarias.

La Ley 29/1991 en su artículo 16.2 trasladó el contenido de la Directiva en relación al concepto de motivo económicamente válido pero sin mencionar tal concepto en su redacción.

Actualmente, la Ley 27/2014, de 27 de noviembre del Impuesto sobre Sociedades (en adelante LIS) recoge explícitamente el concepto de motivo económicamente válido en el Régimen Especial Tributario de las fusiones, escisiones, aportaciones de activos y cambio de domicilio social de una sociedad Europea o una Sociedad Cooperativa Europea de un Estado miembro a otro de la Unión Europea.

El artículo 89.2 LIS dispone que:" No se aplicará el régimen establecido en el presente capítulo cuando la operación realizada tenga como principal objetivo el fraude o la evasión fiscal. En particular, el régimen no se aplicará cuando la operación no se efectúe por motivos económicamente válidos, tales como la reestructuración o la racionalización de las actividades de las entidades que participan en la operación, sino con la mera finalidad de conseguir una ventaja fiscal.

Las actuaciones de comprobación de la Administración tributaria que determinen la inaplicación total o parcial del régimen fiscal especial por aplicación de los dispuesto en el párrafo anterior, eliminarán exclusivamente los efectos de la ventaja fiscal".

\footnotetext{
${ }^{16}$ El artículo 11 de la Directiva 90/434/CE establece una medida anti-abuso aplicable por los Estados miembros de tal forma que un Estado podrá negarse a aplicar el régimen fiscal favorable de la Directiva cuando la operación tenga como principal objetivo o como uno de los principales el fraude o la evasión fiscal. El hecho de que una de la operaciones contempladas en el artículo 1 no se efectúe por motivos económicamente válidos como son la reestructuración o la racionalización de las actividades de las sociedades que participan en la operación puede constituir una presunción de que esta operación tiene como objetivo principal el fraude o la evasión fiscal.
} 
Todas las operaciones de reestructuración fiscal quedan englobadas en el ámbito mercantil pero tienen gran trascendencia tributaria, ya que pueden aflorar importantes plusvalías objeto de gravamen por diferentes impuestos, tanto estatales como locales ${ }^{17}$.

La Ley del Impuesto sobre Sociedades prevé, como se ha mencionado, un régimen fiscal especial para todas aquellas operaciones económicas de reestructuración empresarial entre las que se encuentran principalmente las fusiones, escisiones, aportaciones de ramas de actividad y canje de activos.

La finalidad de este régimen es disminuir el coste fiscal que se devenga como consecuencia de las operaciones de reestructuración fiscal a través de un régimen de diferimiento de las plusvalías, de esta manera, se imprime neutralidad fiscal a todas las operaciones que se acojan a este régimen especial del Impuesto sobre Sociedades.

Sin embargo, la norma del Impuesto sobre Sociedades articula un precepto específico que trata de evitar que las sociedades que pretendan realizar algunas de las operaciones de reestructuración empresarial lo hagan por motivos dirigidos exclusivamente al ahorro fiscal. La Ley del Impuesto sobre Sociedades exige que la operación realizada no tenga como principal objetivo el fraude o la evasión fiscal, dándose esta circunstancia cuando la operación de reestructuración empresarial, en general, se realice para mejorar la situación de las empresas y del personal contratado por ellas en el proceso de reestructuración empresarial.

Esta cláusula específica de prevención del fraude fiscal amplía sus efectos a la evasión fiscal, concepto distante al de elusión fiscal pues en la evasión tributaria se produce una sustracción de la obligación del correspondiente impuesto violando, por lo tanto, una norma legal; siendo sancionadas administrativamente tales conductas e incluso penalmente en los casos más graves. Esta cláusula específica de prevención extiende sus efectos ante cualquier incumplimiento material del obligado tributario en las operaciones de reestructuración societaria.

A sensu contrario, para evitar que los órganos de inspección apliquen el precepto, deben ser las razones económicas y no las puramente fiscales las que inciten a aplicar régimen especial de reestructuración empresarial, por lo tanto, debe existir un motivo que desde el punto de vista económico sea válido ${ }^{18}$.

No obstante, el concepto de "económicamente válido" presenta muchos problemas de interpretación al tratarse de un concepto jurídicamente indeterminado, por lo que serán las resoluciones administrativas y jurisdiccionales las que orienten inicialmente a las sociedades en la aplicación de este régimen especial de diferimiento de las plusvalías desde la perspectiva fiscal.

\footnotetext{
${ }^{17}$ En todas las operaciones de reestructuración societaria aparecen figuras tributarias como el IRPF en sede del socio persona física cuando obtiene plusvalías como consecuencia del canje acciones. El Impuesto sobre Sociedades que grava las plusvalías como consecuencia de una transmisión de activos como consecuencia de una fusión por absorción; además de otros impuestos como el que grava el Incremento del Valor de los Bienes de Naturaleza Urbana e incluso el Impuesto sobre el Valor Añadido como consecuencia de realizar aportaciones no dinerarias por empresarios o profesionales para constituir una sociedad.

${ }^{18}$ Vid STJUE 20/05/2010, en relación con la interpretación del artículo 11.1.A) de la Directiva Europea 90/434/CEE
} 
Hay que matizar que el hecho de que la operación de reestructuración se justifique con un motivo económicamente válido no implica, en absoluto, que no pueda surgir un ahorro fiscal en la operación de reestructuración, lo único que ocurre es que la ventaja fiscal debe ser calificada fiscalmente como accesoria a los efectos económicos perseguidos en la operación societaria.

Si inicialmente realizamos una clasificación de los motivos que no son considerados como válidos para la aplicación del régimen especial de reestructuración empresarial tendríamos que pensar en aquellas operaciones de reestructuración en las cuales se persigue la obtención de un crédito tributario sin añadir riqueza al sector productivo donde la sociedad está domiciliada fiscalmente.

A título ilustrativo, una de las operaciones en la que puede dar lugar a que la Administración tributaria no considere que existe un motivo económicamente válido es cuando se realiza una fusión por absorción y exista bases imponibles negativas por compensar en el patrimonio empresarial de la sociedad absorbida. En este sentido, cuando la sociedad que va a ser absorbida no realiza ninguna actividad, ni tiene un patrimonio empresarial relevante, la DGT considera que la finalidad de la operación es aprovechar la base imponible negativa de la sociedad absorbida sin apreciar motivos económicamente válidos ${ }^{19}$.

No obstante, hay que matizar que puede ser que en una fusión, la sociedad absorbida tenga bases imponibles negativas y sin embargo la operación de reestructuración se base en motivos económicamente válidos. Para ello se necesita que las sociedades que intervengan en la fusión realicen algún tipo de actividad y continúen realizándola tras la fusión, de manera que no se trate de una simple operación de liquidación de la sociedad $^{20}$.

Otra operación que es muy común en las reestructuraciones societarias, son las escisiones de empresas, que desde un punto de vista mercantil supone la separación del patrimonio de una sociedad de tal manera que constituyan distintas ramas de actividad con un funcionamiento independiente o bien puede ser que el patrimonio empresarial escindido pueda ser absorbido por otro grupo empresarial.

En este tipo de reestructuración empresarial se han considerado como motivos económicamente válidos la separación de activos y pasivos afectos a un negocio en particular de otros activos, por ejemplo, en ocasiones y por mor de reducir ciertos riesgos del mercado es aceptable separar del patrimonio empresarial global, el patrimonio inmobiliario de una empresa del resto de activos. Con ese tipo de escisión, la empresa ganaría seguridad en el mercado así como facilitaría su acceso al crédito bancario $^{21}$.

También es habitual que las operaciones de escisión societarias se realicen con el objeto de reorganizar un grupo empresarial para más adelante planificar la futura transmisión del patrimonio empresarial escindido a los descendientes del titular del patrimonio empresarial. En determinadas estrategias fiscales de tipo sucesorio el titular del

\footnotetext{
${ }^{19}$ Vid Resol DGT n ${ }^{\circ}$ V0072-14

${ }^{20}$ Vid Resol TEAC 27/07/2006 y DGT 29/04/2014

${ }^{21}$ Vid Resol DGT 20/01/2014
} 
patrimonio empresarial acude a la segregación del patrimonio empresarial para crear nuevas empresas de modo que, vía sucesión hereditaria cada causahabiente heredaría una sociedad mercantil ${ }^{22}$.

En sentido contrario, la Administración tributaria no considera motivos económicamente válidos en las escisiones de empresas cuando la escisión se considera como un verdadero mecanismo en la separación de socios ya sea como consecuencia de la propia escisión o como consecuencia de las operaciones realizadas por éstos, una vez materializada la escisión.

\section{Conclusiones}

El conflicto en la aplicación de la norma tributaria se diferencia del concepto de fraude de ley en el Derecho tributario en el sentido de que ya no se admite una interpretación de la norma cuando los obligados tributarios realicen una estrategia fiscal agresiva utilizando actos que impliquen un abuso de las formas jurídicas. En este sentido, el legislador español tiene en cuenta los aspectos subjetivos del contribuyente que trata de eludir el impuesto utilizando una norma de cobertura con el único fin de ahorrar impuestos.

Actualmente, el conflicto en la aplicación de la norma tributaria es susceptible en determinados casos de ser sancionado por la Administración tributaria. La nueva regulación del conflicto en la aplicación de la norma tributaria tiene como función disuadir determinados comportamientos tendentes al abuso de las formas jurídicas por los obligados tributarios.

Las operaciones de reestructuración empresarial deben hacerse por un motivo que desde un punto de vista económico sea válido, como por ejemplo, aumentar o mantener la plantilla de trabajadores de las empresas que hayan experimentado las operaciones de reestructuración. En ningún caso, se considerará la compensación de las bases imponibles negativas de una sociedad que va a ser absorbida como un motivo económicamente válido que justifique el régimen fiscal especial de reestructuración empresarial.

\section{Bibliografía}

CALVO VERGEZ ,J.(2007) “Cláusula antiabuso versus fraude de ley: ¿dos conceptos semejantes en la Ley General Tributaria?” Crónica Tributaria; nº 123.

HERRERA MOLINA, PM. (1999) "Nuevas perspectivas del fraude de ley en materia tributaria" Quincena Fiscal.

PALAO TABOADA; C (1998). "Existe fraude a la ley tributaria" Revista de Tributación y Contabilidad, $\mathrm{n}^{\circ} 182$.

PÉREZ ROLLO, F y AGUALLO AVILÉS, A (1996). Comentarios a la reforma de la Ley General Tributaria; Aranzadi.

\footnotetext{
${ }^{22}$ Vid Resol DGT 19/11/2014
} 